\title{
1. Scaled boundary FEM solution of short-crested wave diffraction by a vertical cylinder
}

\section{Abstract}

7 The scaled boundary finite-element method (SBFEM) is a novel semi-analytical method 8 developed in the elasto-statics and elasto-dynamics areas that has the advantages of com9 bining the finite-element method with the boundary-element method. The SBFEM method weakens the governing differential equation in the circumferential direction and solves the weakened equation analytically in the radial direction. It has the inherent advantage of solving the unbounded fluid dynamic problem. In this paper, the boundary-value problem composed of short-crested waves diffracted by a vertical circular cylinder is solved by SBFEM. Only the cylinder boundary is discretized with curved surface finite-elements on the circumference of the cylinder, while the radial differential equation is solved completely analytically. The computation of the diffraction force based on the present SBFEM solution demonstrates a high accuracy achieved with a small number of surface finite-elements. The method can be extended to solve more complex wave-structure interaction problems resulting in direct engineering applications.

Key words: scaled boundary finite-element method, short-crested wave, wave diffraction, unbounded domain, vertical cylinder 
Studies on ocean surface waves are generally focused on two-dimensional (2D) waves. In reality, however, the ocean waves are more complex. The short-crested wave is a common $3 \mathrm{D}$ wave model which describes waves generated by winds blowing across the surface of the ocean. It also commonly arises, for example, from the oblique interaction of two travelling plane waves or intersecting swell waves, from the reflection of waves at non-normal incidence off a vertical seawall or a breakwater, as well as from diffraction about the surface boundaries of a structure of finite length [6]. Such waves are of paramount importance in an engineering design. Unlike the plane waves propagating in a single direction, and the standing waves fluctuating vertically in a confined region, short-crested waves can be doubly periodic in two horizontal directions, one in the direction of propagation and the other normal to it [15].

The interaction problem of water waves with a large vertical circular cylinder has been widely investigated both numerically and experimentally in the past due to its theoretical and practical importance, especially to ocean engineers. Diffraction effects become more important when the dimension of the structure is large compared to the wave length. As one of the pioneers in wave-structure interaction research, MacCamy and Fuchs [10] presented an analytical solution for linear plane waves diffracted by a large vertical cylinder in intermediate water depths and their solution was later validated by Chakrakarti and Tam's [2] experiment. Chakrabarti and Tam [2] revealed that the linear diffraction solution is reasonably accurate at least for $H / h \leq 0.25$ ( $H$ is wave height and $h$ is water depth) and a range of $k a$

* Corresponding author. Tel.: +61 755529093; fax: +61 755528065. Email address: 1 .tao@griffith.edu.au (Longbin Tao $\left.{ }^{1}\right)$. 
( $k$ is wave number and $a$ is cylinder radius) between 0 and 3. Since then, many numerical and experimental results have been reported on the plane wave diffraction $[1,7,9,12-14]$. However, studies on the short-crested wave diffraction are very limited in the literature.

Zhu [17] solved the diffraction problem for a circular cylinder in short-crested waves using linear potential flow theory and found that the pressure distribution and water run-up on the cylinder were quite different from those of plane incident waves. Their patterns become very complex as $k a$ becomes large. The hydrodynamic forces on the cylinder become larger as the incident waves become less shortcrested. Based on the boundary-element method solution, Zhu and Moule [18] examined the dependence of the wave diffraction force for a given cylinder on the wave number and found that the wave loads induced by short-crested waves can be larger than those induced by plane waves for the same total wave number. Zhu and Satravaha [19] further presented the second-order diffraction solution of shortcrested waves. However, the full analytical results are very complex and need validation.

Recently, a new semi-analytical method, called scaled boundary finite-element method (SBFEM) has been successfully applied to soil-structure interaction problems. Combining the advantages of finite-element and boundary-element methods, the approach discretizes only the boundary with surface finite-elements. It then transforms the governing partial differential equations to ordinary differential equations, and solves them analytically. The method represents singularities and unbounded domains accurately and efficiently compared to the complete finite-element method and needs no fundamental solution as for the boundary-element method. Fewer elements are required to obtain very accurate results [16]. Deeks and Cheng [4] applied SBFEM to an uniform potential flow around obstacles and revealed its inherent ad- 
vantages to model unbounded fluid problems as well as the singular points in the near field of bluff obstacles. $\mathrm{Li}$, et al [8] attempted to solve the problem of plane wave diffraction by a vertical cylinder using SBFEM, with only limited success in obtaining semi-analytical solution for high frequency waves. For low frequency waves, other numerical methods, such as, the Runge-Kutta scheme needs to be implemented to solve the radial differential equation. Such compromised approach significantly diminishes, to a large extend, the advantages of SBFEM exhibited in dealing with bounded fluid domain problems.

In this paper, linear short-crested wave diffraction by a large vertical circular cylinder in an unbounded domain is solved by SBFEM. The radial differential equation is solved fully analytically. Only a few finite elements discretized on the circumference of the cylinder are shown to be sufficient to obtain accurate results. Excellent agreements between the present SBFEM results and the analytical solutions of Zhu [17], including wave run-up, diffraction force and other hydrodynamic force coefficients are achieved. The method described in this paper can be easily extended to apply to more complex practical engineering problems due to its excellent computational efficiency and accuracy, which appears to be the significant limitation associated with most of the existing numerical models for wave-structure interaction.

\section{Theoretical Consideration}

\subsection{Problem definition}

Consider a monochromatic short-crested wave train propagating in the direction of the positive $x$ axis. A fixed vertical cylinder extends from the sea bottom to 


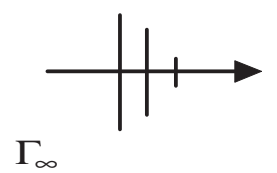

$\Omega$
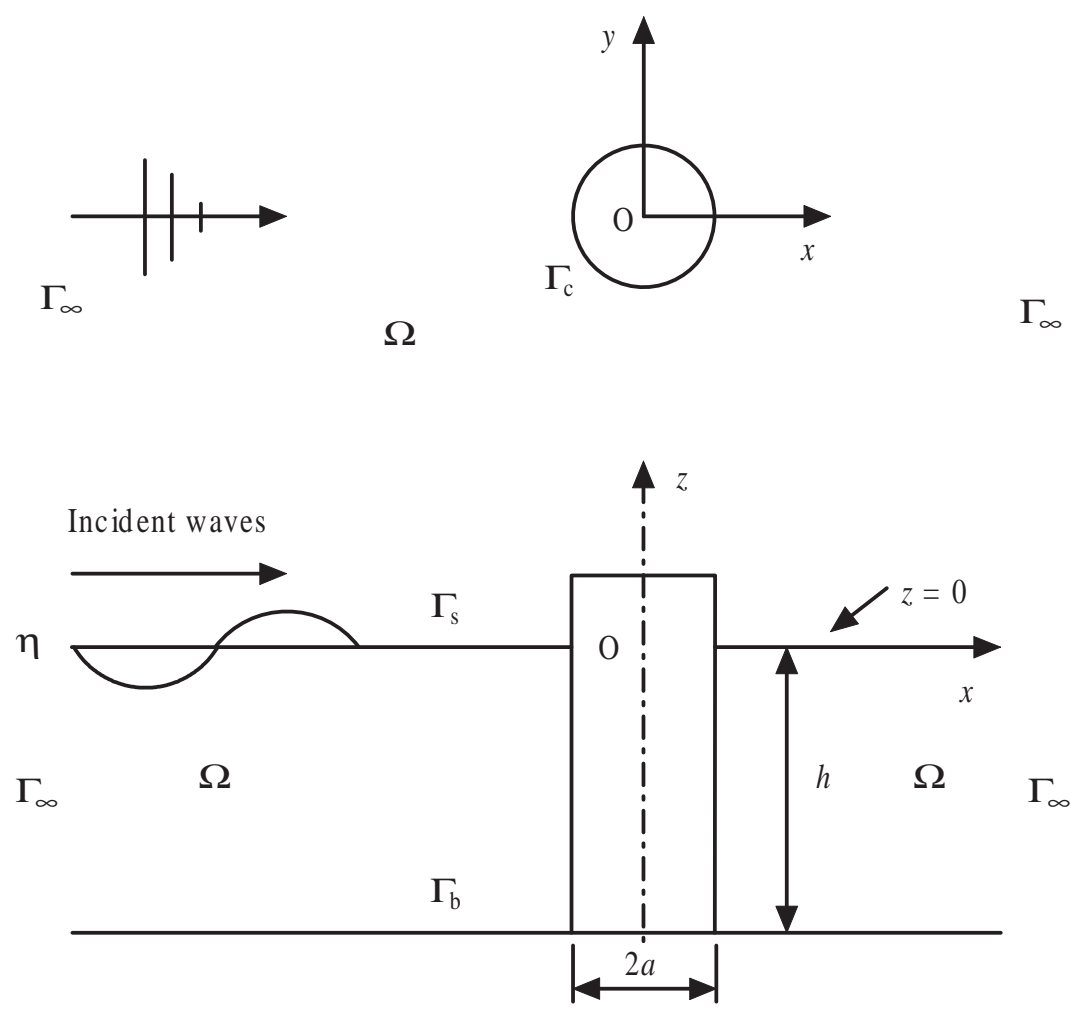

Fig. 1. Problem definition.

above the free surface of the ocean along $z$ axis. The origin is placed at the centre of the cylinder on the mean water surface (Fig. 1). The total velocity potential, the velocity potential of incident wave, the velocity potential of scattered wave, total wave number, wave number in $x$ direction, wave number in $y$ direction, wave frequency, water depth, the amplitude of incident wave, the cylinder radius, the time, and the gravitational acceleration are denoted respectively as $\Phi, \Phi_{I}, \Phi_{S}, k$, $k_{x}, k_{y}, \omega, h, A, a, t$, and $g$.

Assuming the fluid to be inviscid, incompressible and the flow to be irrotational, the fluid motion can be described by a velocity potential $\Phi$ satisfying the Laplace equation

$$
\nabla^{2} \Phi=0 \quad \text { in } \quad \Omega
$$


subject to the linearized combined free surface boundary condition

$$
\Phi_{, t t}+g \Phi_{, z}=0 \quad \text { at } \quad z=0
$$

and the bottom condition

$$
\Phi_{, z}=0 \quad \text { at } \quad z=-h,
$$

where

$$
\begin{aligned}
\Phi(x, y, z, t) & =\phi(x, y) Z(z) e^{-i \omega t}, \\
\Phi_{I}(x, y, z, t) & =\phi^{I}(x, y) Z(z) e^{-i \omega t}, \\
\Phi_{S}(x, y, z, t) & =\phi^{S}(x, y) Z(z) e^{-i \omega t},
\end{aligned}
$$

$$
Z(z)=\frac{\cosh k(z+h)}{\cosh k h} .
$$

110 This procedure leads to the sea bottom condition being automatically satisfied, and the linear free surface boundary condition is satisfied using the following dispersion relationship 


$$
\omega^{2}=g k \tanh k h
$$

113 Thus, the problem reduces to two-dimensional at the free surface. The function $\phi^{S}(x, y)$ is governed by the Helmholtz equation with the boundary condition at the interface of fluid and structure, and the radiation condition at infinity, the so-called 116 Sommerfeld condition:

$$
\nabla^{2} \phi^{S}+k^{2} \phi^{S}=0
$$

$$
\phi_{, n}^{S}=-\phi_{, n}^{I} \quad \text { on } \quad r=a,
$$

$$
\lim _{k r \rightarrow \infty}(k r)^{1 / 2}\left(\phi_{, r}^{S}-i k \phi^{S}\right)=0
$$

117 where $r$ is the radial axis, $i=\sqrt{-1}$, and $n$ denotes the normal to the boundary.

118 The velocity potential of the linear short-crested incident wave travelling in the 119 positive $x$ direction is given by the real part of [5]:

$$
\Phi_{I}=-\frac{i g A}{\omega} Z(z) e^{i\left(k_{x} x-\omega t\right)} \cos \left(k_{y} y\right),
$$
and the relationship of the total velocity potential, with the scattered, and the incident wave velocity potentials is

$$
\Phi=\Phi_{I}+\Phi_{S}, \quad \phi=\phi^{I}+\phi^{S} .
$$




$$
\begin{gathered}
\mathbf{v}=\nabla \Phi, \\
\eta=\frac{i \omega}{g} \phi, \\
p=-\rho \Phi_{, t},
\end{gathered}
$$

where $\rho$ is the mass density of water.

\subsection{Scaled boundary finite-element method}

As shown in Fig. 1, (9) is valid in the whole fluid domain $\Omega$ with the fluid-structure interface $\Gamma_{c}$ and infinity boundary $\Gamma_{\infty}$. If the velocity boundary is defined by $\Gamma_{v}$, we have

$$
\phi_{, n}^{S}=\bar{v}_{n}, \quad \text { on } \quad \Gamma_{v},
$$

where the overbar denotes a prescribed value. To derive a finite-element approximation, the weighted residual technique is applied by multiplying a weighting function $w$ to (9) and (17), and integrating over the domain and the boundary. 


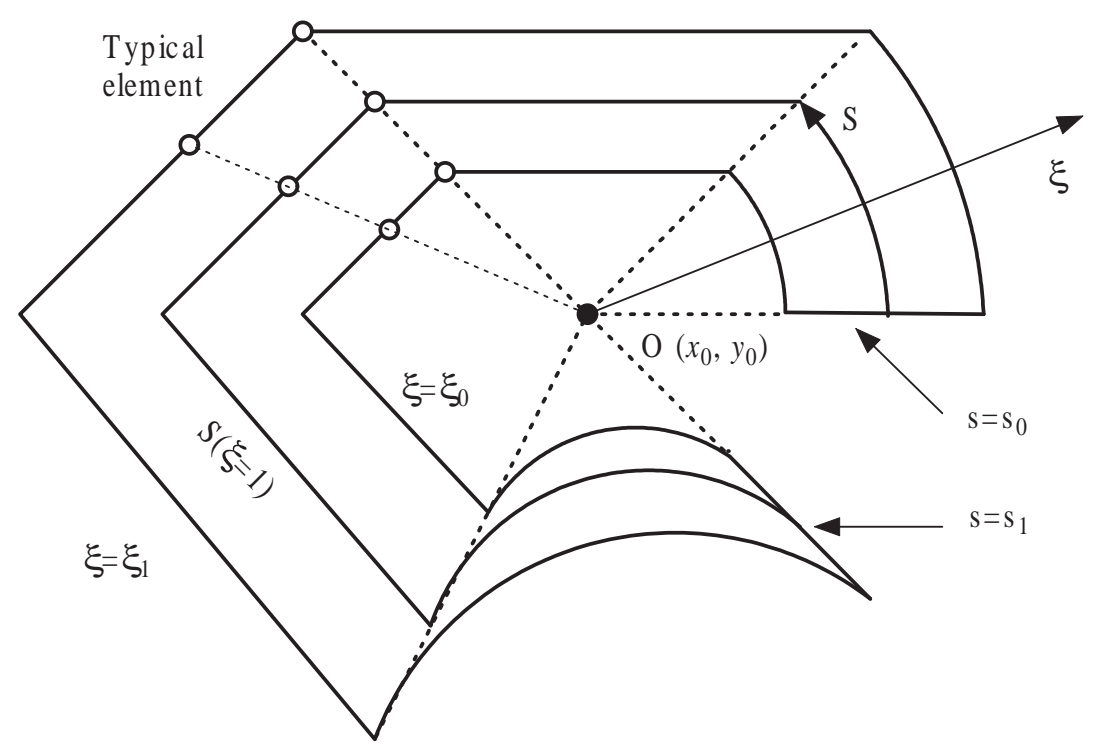

Fig. 2. The coordinate definition of SBFEM.

$$
\int_{\Omega} \nabla^{T} w \nabla \phi^{S} d \Omega-\int_{\Omega} w k^{2} \phi^{S} d \Omega-\oint_{\Gamma} w \bar{v}_{n} d \Gamma=0
$$

$$
x=x_{0}+\xi x_{s}(s), \quad y=y_{0}+\xi y_{s}(s) .
$$

By employing SBFEM, an approximate solution of $\phi^{S}$ is sought as 


$$
\phi_{A}(\xi, s)=\mathbf{N}(s) \mathbf{a}(\xi)
$$

where

$$
\nabla=\mathbf{b}_{1}(s) \frac{\partial}{\partial \xi}+\frac{1}{\xi} \mathbf{b}_{2}(s) \frac{\partial}{\partial s}
$$

where $\mathbf{b}_{1}(s)$ and $\mathbf{b}_{2}(s)$ are dependent only on the boundary definition

$$
\mathbf{b}_{1}(s)=\frac{1}{|J|}\left\{\begin{array}{c}
y_{s}(s)_{, s} \\
-x_{s}(s)_{, s}
\end{array}\right\}, \quad \mathbf{b}_{2}(s)=\frac{1}{|J|}\left\{\begin{array}{c}
-y_{s}(s) \\
x_{s}(s)
\end{array}\right\}
$$

and $|J|$ is the Jacobian at the boundary

$$
|J|=x_{s}(s) y_{s}(s)_{, s}-y_{s}(s) x_{s}(s)_{, s} .
$$

From (14) and (21), the approximate velocity can be expressed as

$$
\mathbf{v}_{A}(\xi, s)=\mathbf{B}_{1}(s) \mathbf{a}(\xi)_{, \xi}+\frac{1}{\xi} \mathbf{B}_{2}(s) \mathbf{a}(\xi),
$$




$$
\mathbf{B}_{1}(s)=\mathbf{b}_{1}(s) \mathbf{N}(s), \quad \mathbf{B}_{2}(s)=\mathbf{b}_{2}(s) \mathbf{N}(s)_{, s} .
$$

155 The weighting function $w$ can be chosen as the same shape function as (20) by 156 Galerkin approach

$$
w(\xi, s)=\mathbf{N}(s) \mathbf{w}(\xi)=\mathbf{w}(\xi)^{T} \mathbf{N}(s)^{T}
$$

157 Using (20), (21), (25) and (26), (18) becomes

$$
\begin{aligned}
& \int_{\Omega}\left[\mathbf{B}_{1}(s) \mathbf{w}(\xi)_{, \xi}+\frac{1}{\xi} \mathbf{B}_{2}(s) \mathbf{w}(\xi)\right]^{T}\left[\mathbf{B}_{1}(s) \mathbf{a}(\xi)_{, \xi}+\frac{1}{\xi} \mathbf{B}_{2}(s) \mathbf{a}(\xi)\right] d \Omega \\
& -\int_{\Omega} k^{2} \mathbf{w}(\xi)^{T} \mathbf{N}(s)^{T} \mathbf{N}(s) \mathbf{a}(\xi) d \Omega-\oint_{\Gamma} \mathbf{w}(\xi)^{T} \mathbf{N}(s)^{T} \bar{v}_{n} d \Gamma=0,
\end{aligned}
$$

158 where the incremental volume is $[4,16]$

$$
d \Omega=|J| \xi d \xi d s
$$

159 Introducing the coefficient matrices

$$
\begin{aligned}
\mathbf{E}_{0} & =\int_{S} \mathbf{B}_{1}(s)^{T} \mathbf{B}_{1}(s)|J| d s, \\
\mathbf{E}_{1} & =\int_{S} \mathbf{B}_{2}(s)^{T} \mathbf{B}_{1}(s)|J| d s, \\
\mathbf{E}_{2} & =\int_{S} \mathbf{B}_{2}(s)^{T} \mathbf{B}_{2}(s)|J| d s, \\
\mathbf{M}_{0} & =\int_{S} \mathbf{N}(s)^{T} \mathbf{N}(s)|J| d s, \\
\mathbf{F}_{s}(\xi) & =\mathbf{N}\left(s_{0}\right)^{T}\left(-\bar{v}_{n}\left(\xi, s_{0}\right)\right)\left|J\left(s_{0}\right)\right|+\mathbf{N}\left(s_{1}\right)^{T}\left(-\bar{v}_{n}\left(\xi, s_{1}\right)\right)\left|J\left(s_{1}\right)\right| .
\end{aligned}
$$


The above integrals (29)-(32) can be computed element by element and assembled together for the entire boundary. Expanding (27) and integrating the terms containing $\mathbf{w}(\xi)_{, \xi}$ by parts with respect to $\xi$ using Green's theorem leads to

$$
\begin{aligned}
& \mathbf{w}\left(\xi_{1}\right)^{T}\left[\mathbf{E}_{0} \xi_{1} \mathbf{a}\left(\xi_{1}\right)_{, \xi}+\mathbf{E}_{1}^{T} \mathbf{a}\left(\xi_{1}\right)-\int_{S} \mathbf{N}(s)^{T}\left(\bar{v}_{n}\left(\xi_{1}, s\right)\right) \xi_{1} d s\right] \\
- & \mathbf{w}\left(\xi_{0}\right)^{T}\left[\mathbf{E}_{0} \xi_{0} \mathbf{a}\left(\xi_{0}\right)_{, \xi}+\mathbf{E}_{1}^{T} \mathbf{a}\left(\xi_{0}\right)+\int_{S} \mathbf{N}(s)^{T}\left(\bar{v}_{n}\left(\xi_{0}, s\right)\right) \xi_{0} d s\right] \\
- & \int_{\xi_{0}}^{\xi_{1}} \mathbf{w}(\xi)^{T}\left[\mathbf{E}_{0} \xi \mathbf{a}(\xi)_{, \xi \xi}+\left(\mathbf{E}_{0}+\mathbf{E}_{1}^{T}-\mathbf{E}_{1}\right) \mathbf{a}(\xi)_{, \xi}-\mathbf{E}_{2} \frac{1}{\xi} \mathbf{a}(\xi)+k^{2} \xi \mathbf{M}_{0} \mathbf{a}(\xi)-\mathbf{F}_{s}(\xi)\right] d \xi \\
= & 0 .
\end{aligned}
$$

To satisfy all sets of weighting function $\mathbf{w}(\xi)$, the following conditions must be satisfied:

$$
\begin{gathered}
\mathbf{E}_{0} \xi_{1} \mathbf{a}\left(\xi_{1}\right)_{, \xi}+\mathbf{E}_{1}^{T} \mathbf{a}\left(\xi_{1}\right)=\int_{S} \mathbf{N}(s)^{T}\left(\bar{v}_{n}\left(\xi_{1}, s\right)\right) \xi_{1} d s, \\
\mathbf{E}_{0} \xi_{0} \mathbf{a}\left(\xi_{0}\right)_{, \xi}+\mathbf{E}_{1}^{T} \mathbf{a}\left(\xi_{0}\right)=-\int_{S} \mathbf{N}(s)^{T}\left(\bar{v}_{n}\left(\xi_{0}, s\right)\right) \xi_{0} d s, \\
\mathbf{E}_{0} \xi^{2} \mathbf{a}(\xi)_{, \xi \xi}+\left(\mathbf{E}_{0}+\mathbf{E}_{1}^{T}-\mathbf{E}_{1}\right) \xi \mathbf{a}(\xi)_{, \xi}-\mathbf{E}_{2} \mathbf{a}(\xi)+k^{2} \xi^{2} \mathbf{M}_{0} \mathbf{a}(\xi)=\xi \mathbf{F}_{s}(\xi) .
\end{gathered}
$$

The equation (37) is the so-called scaled boundary finite-element equation. By introducing the shape function, the Helmholtz equation has been weakened in the circumferential direction, so that the governing partial differential equation is transformed to an ordinary matrix differential equation in radial direction. The rank of matrices $\mathbf{E}_{0}, \mathbf{E}_{1}, \mathbf{E}_{2}, \mathbf{M}_{0}$ and vector $\mathbf{a}(\xi)$ is $m$ (where $m$ is the number of nodes in the curve $S$ ). In the present study, the side-faces coincide so that the flow across the side-faces is equal and opposite, thus the term $\mathbf{F}_{s}(\xi)$ vanishes. Therefore, the final 


\subsection{Solution technique}

For a vertical circular cylinder, we have,

$$
x_{s}(s)=a \cos (s / a), \quad y_{s}(s)=a \sin (s / a) .
$$

From (19), (22), (23), (25) and (29)-(32), $x_{s}(s)_{, s}, y_{s}(s)_{, s}, \mathbf{b}_{1}(s), \mathbf{b}_{2}(s),|J|, \mathbf{B}_{1}(s)$, $\mathbf{B}_{2}(s), \mathbf{E}_{0}, \mathbf{E}_{1}, \mathbf{E}_{2}$, and $\mathbf{M}_{0}$ can be calculated accordingly. The following relations hold:

$$
\mathbf{E}_{1}=0 \cdot \mathbf{I}, \quad \mathbf{E}_{0}^{-1} \mathbf{M}_{0}=a^{2} \mathbf{I},
$$

where $\mathbf{I}$ is the identity matrix of rank $m$.

Using (39), pre-multiplying both sides of (37) by $\mathbf{E}_{0}^{-1}$ and simplifying, we have

$$
\zeta^{2} \mathbf{a}(\zeta)_{, \zeta \zeta}+\zeta \mathbf{a}(\zeta)_{, \zeta}-\mathbf{E}_{0}^{-1} \mathbf{E}_{2} \mathbf{a}(\zeta)+\zeta^{2} \mathbf{a}(\zeta)=0
$$




$$
\zeta=k a \xi
$$

186

$$
\sum_{j=1}^{m}\left(\mathbf{E}_{0}^{-1} \mathbf{E}_{2}-r_{j}^{2} \mathbf{I}\right) \mathbf{T}_{j} \cdot c_{j} H_{r_{j}}(\zeta)=0
$$

195 For any $c_{j} H_{r_{j}}(\zeta),(45)$ yields

$$
\left(\mathbf{E}_{0}^{-1} \mathbf{E}_{2}-r_{j}^{2} \mathbf{I}\right) \mathbf{T}_{j}=0 .
$$


Define

$$
\mathbf{E}_{0} k a \sum_{j=1}^{m} c_{j} H_{r_{j}}^{\prime}(k a) \mathbf{T}_{j}=-\left[\int_{S} \mathbf{N}(s)^{T} \mathbf{N}(s) d s\right] \overline{\mathbf{v}}_{n}^{S},
$$

where $\overline{\mathbf{v}}_{n}^{S}$ is the vector of nodal normal velocity of scattered wave on the body boundary.

For a circular cylinder,

$$
\mathbf{E}_{0}=\frac{1}{a} \int_{S} \mathbf{N}(s)^{T} \mathbf{N}(s) d s
$$

$$
\begin{aligned}
\mathbf{T} & =\left[\mathbf{T}_{1}, \mathbf{T}_{2}, \cdots, \mathbf{T}_{m}\right], \\
\mathbf{C} & =\left[c_{1}, c_{2}, \cdots, c_{m}\right]^{T}, \\
\mathbf{H} & =\operatorname{diag}\left[H_{r_{1}}(k a \xi), H_{r_{2}}(k a \xi), \cdots, H_{r_{m}}(k a \xi)\right], \\
\mathbf{H}_{d} & =\operatorname{diag}\left[H_{r_{1}}^{\prime}(k a), H_{r_{2}}^{\prime}(k a), \cdots, H_{r_{m}}^{\prime}(k a)\right],
\end{aligned}
$$
the main diagonal; then $\mathbf{C}$ can be solved from (47) as 


$$
\mathbf{C}=-\frac{1}{k} \mathbf{H}_{d}^{-1} \mathbf{T}^{-1} \overline{\mathbf{v}}_{n}^{S}
$$

207 The solution of $\mathbf{a}(\zeta)$ is obtained as

$$
\mathbf{a}(\zeta)=\mathbf{T H C}=-\frac{1}{k} \mathbf{T} \mathbf{H}_{h} \mathbf{T}^{-1} \overline{\mathbf{v}}_{n}^{S},
$$

where $\mathbf{H}_{h}=\operatorname{diag}\left[H_{r_{1}}(k a \xi) / H_{r_{1}}^{\prime}(k a), H_{r_{2}}(k a \xi) / H_{r_{2}}^{\prime}(k a), \cdots, H_{r_{m}}(k a \xi) / H_{r_{m}}^{\prime}(k a)\right]$. Using (4), (5), (10), (12) and (17), $\overline{\mathbf{v}}_{n}^{S}$ can be easily determined on the body boundary. From (20) and (54), the approximation of scattered velocity potential can be obtained in the whole domain. The total velocity potential can then be calculated by (6) and (13). All the other physical properties such as elevation, pressures can be calculated accordingly.

In this paper, the boundary $\Gamma_{c}$ is discretized into $N$ three-noded quadratic elements in circumferential direction. Due to the symmetry of the physical problem, only half of the circumference is discretized.

The total force, per unit length in the direction of wave propagation is

$$
\frac{d F_{x}}{d z}=-a \int_{0}^{2 \pi} p \cdot \cos (\theta) d \theta=2 \pi a R\left(k_{x}, k_{y}, k, a\right) \cdot \rho g A \cdot Z(z) e^{-i \omega t}
$$

where the function $R\left(k_{x}, k_{y}, k, a\right)$ is a dimensionless parameter of $\frac{d F_{x}}{d z}$ without the term $\rho g A \cdot Z(z) e^{-i \omega t}$.

The function $R\left(k_{x}, k_{y}, k, a\right)$ determines the first-order total horizontal force in $x$ direction on the cylinder, $F_{x}$, which can be obtained by integrating Eq. (55) with respect to $z$, 


$$
F_{x}=\int_{-h}^{0} \frac{d F_{x}}{d z} d z=2 \pi a R\left(k_{x}, k_{y}, k, a\right) \cdot \rho g A e^{-i \omega t} \cdot \tanh (k h) / k
$$

Following traditional concept, the component of force per unit of wave height in phase with the particle acceleration of the incident waves is called an effective inertia coefficient $C_{M}$ and that in phase with the particle velocity is termed an effective linear drag coefficient $C_{D}$. Then the force is written as follows:

$$
\operatorname{Re}\left(\frac{d F_{x}}{d z}\right)=\rho \pi a^{2}\left(C_{M} \dot{U}+\omega C_{D} U\right)
$$

where $U$ is the velocity of the incident waves at the origin of the cylinder in its absence.

Using (12), (55) and (57), we have

$$
C_{M}=-\frac{2 R_{i}}{k_{x} a}, \quad C_{D}=\frac{2 R_{r}}{k_{x} a},
$$

where $R_{r}$ and $R_{i}$ are the real and imaginary parts of $R\left(k_{x}, k_{y}, k, a\right)$.

\section{Results and Discussion}

The present SBFEM scheme is verified against (1) analytical solutions for plane waves [11]; and (2) analytical solutions for short-crested waves [17]. Figs. 3-6 are the comparisons of wave run-up between the present SBFEM results and the analytical solutions shown in [11]. As can be seen in the figures, for small $k a(=0.5)$, only two elements discretized over the cylinder boundary are sufficient to yield good agreement between the present SBFEM result and the analytical solution. As 


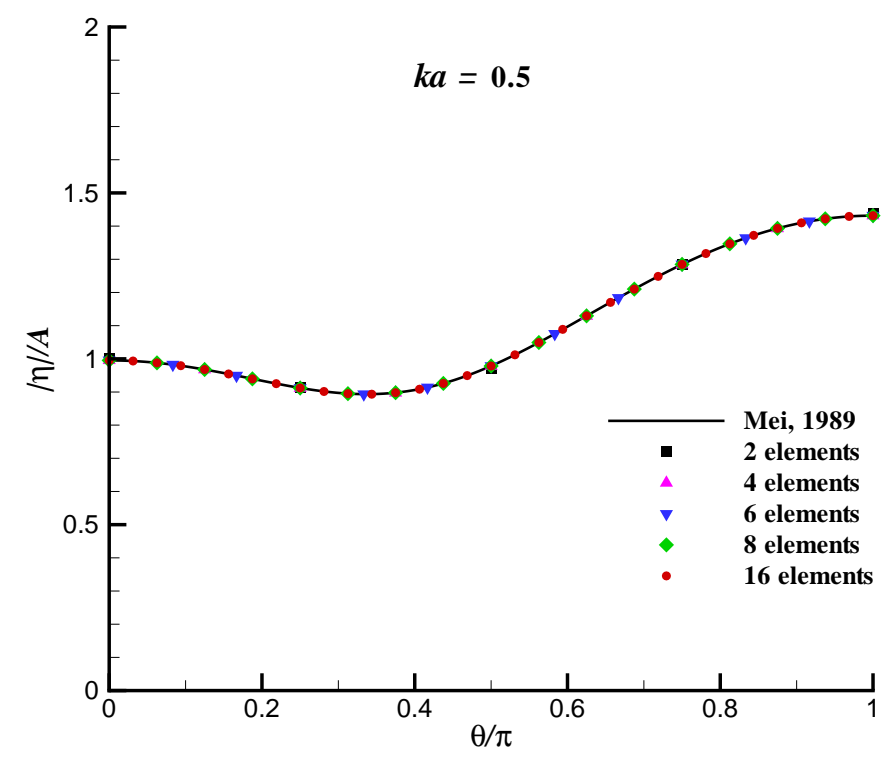

Fig. 3. The run-up of progressive wave on a circular cylinder extending the entire sea depth $(k a=0.5)$.

$k a$ increases from 0.5 to 5.0, the convergence of the SBFEM scheme is clearly seen as the number of elements is increased. Even at $k a=5.0$, satisfactory numerical results were obtained when merely 6 elements were used.

The run-up on the circular cylinder due to short-crested waves is shown in Figs. 711. In these examples the total incident wave number is the same. Again, excellent agreement with the solutions of Zhu [17] is found where the SBFEM results with only 4 elements gave almost identical solutions to the analytical. Fig. 7 shows that the largest run-up occurs near the front face of the cylinder $(\theta / \pi \approx 1)$ when the incident waves have infinite wavelength $\left(k_{y}=0\right)$ in the direction perpendicular to the wave propagation, i.e., plane waves. The other extreme case, shown in Fig. 11, represents standing waves when the incident waves have infinite wavelength $\left(k_{x}=0\right)$ in the direction of propagation. In order to show the difference of the run-up on the cylinder between the plane waves and short-crested waves, Fig. 12 is 


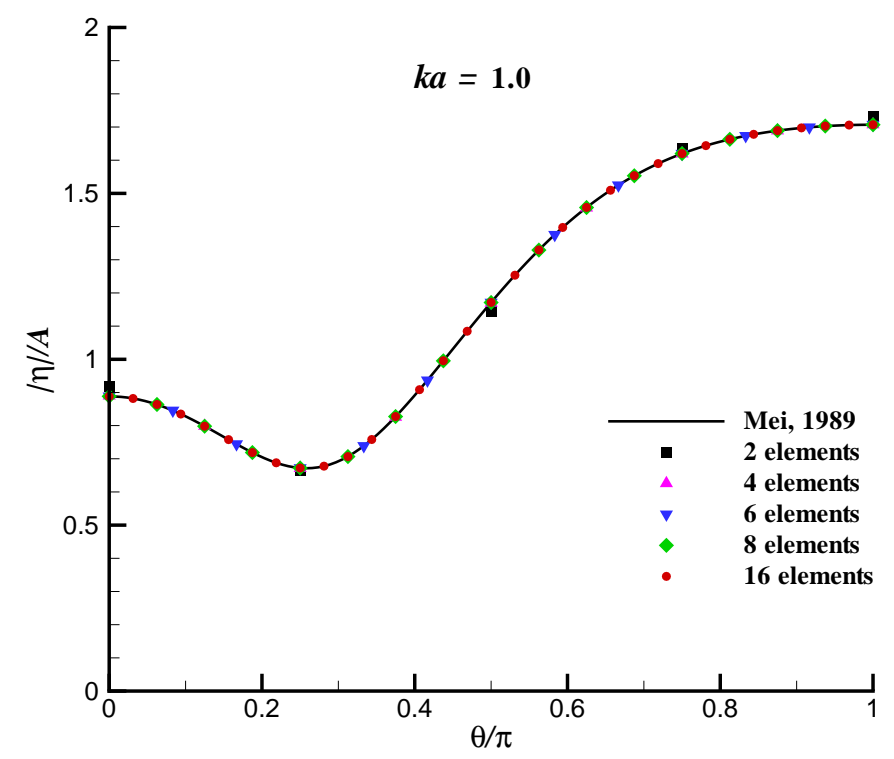

Fig. 4. The run-up of progressive wave on a circular cylinder extending the entire sea depth $(k a=1.0)$.

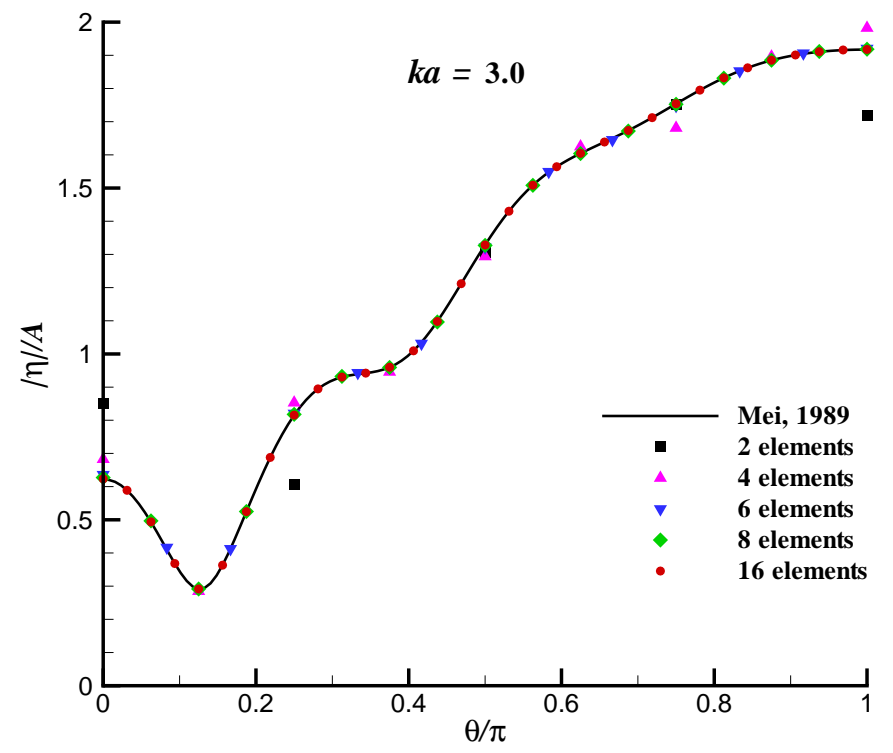

Fig. 5. The run-up of progressive wave on a circular cylinder extending the entire sea depth $(k a=3.0)$. 


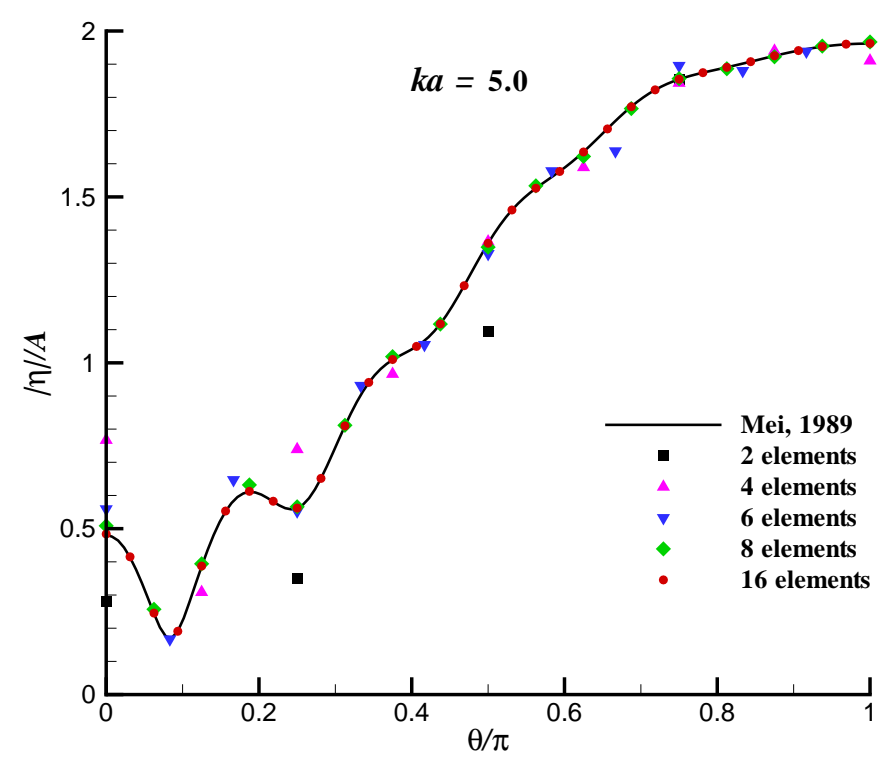

Fig. 6. The run-up of progressive wave on a circular cylinder extending the entire sea depth $(k a=5.0)$.

an overlay of the plots of the present SBFEM results with 4 finite elements shown in Figs. 7-11. It is observed in Fig. 12 that the elevation on the cylinder surface is quite different when the incident waves become short-crested. As the incident wave becomes more short-crested, the elevation near the front face of the cylinder $(\theta / \pi \approx 1)$ decreases monotonically, however, no similar attribute is observed on the lee side of the cylinder $(\theta / \pi \approx 0)$.

Table 1 shows the comparisons of the effective inertia, and the effective linear drag coefficients and total forces. Excellent computational efficiency and accuracy of the present numerical scheme are further demonstrated by examining the hydrodynamic forces. It is apparent that only four elements can lead to quite accurate results while six elements produce almost identical results as the analytical solution [17]. As pointed out by Zhu [17], both the effective inertia coefficient, $C_{M}$, and the effective drag coefficient, $C_{D}$, are generally functions of $k_{x}$ and $k_{y}$. However, 


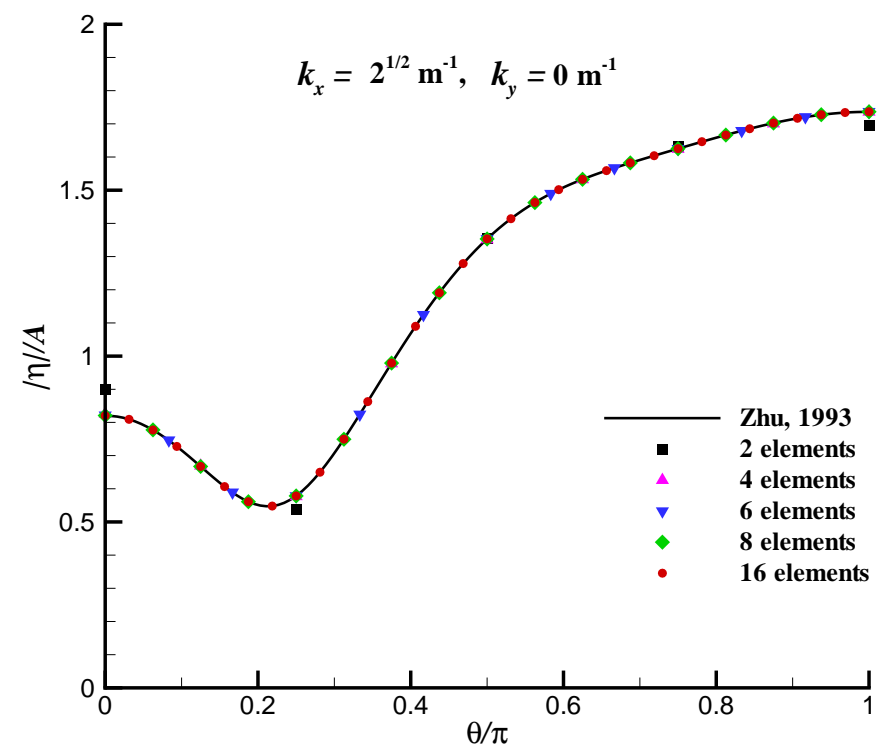

Fig. 7. Comparison of short-crested wave run-up on a cylinder with radius $a=1.0 \mathrm{~m}$ and total incident wave number $k=\sqrt{2} \mathrm{~m}^{-1}\left(k_{x}=\sqrt{2} \mathrm{~m}^{-1}, k_{y}=0 \mathrm{~m}^{-1}\right)$.

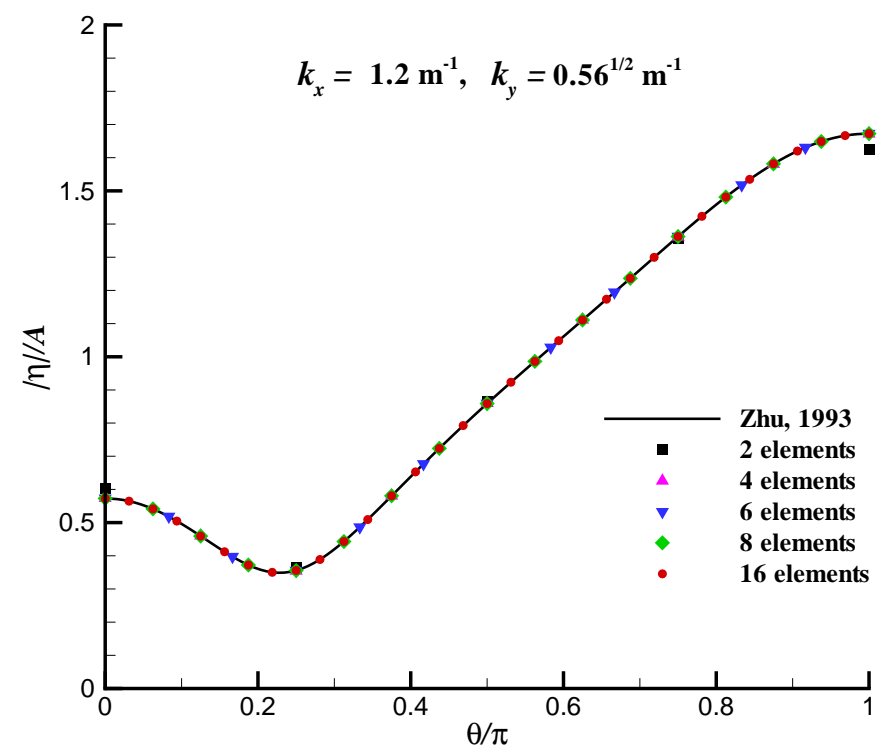

Fig. 8. Comparison of short-crested wave run-up on a cylinder with radius $a=1.0 \mathrm{~m}$ and total incident wave number $k=\sqrt{2} \mathrm{~m}^{-1}\left(k_{x}=1.2 \mathrm{~m}^{-1}, k_{y}=\sqrt{0.56} \mathrm{~m}^{-1}\right)$. 


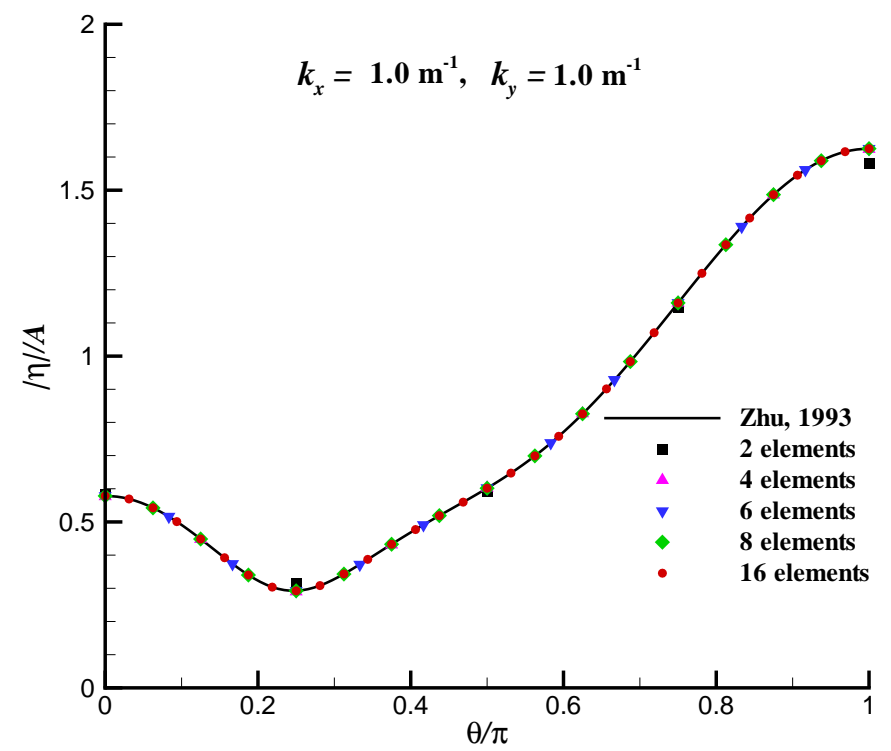

Fig. 9. Comparison of short-crested wave run-up on a cylinder with radius $a=1.0 \mathrm{~m}$ and total incident wave number $k=\sqrt{2} \mathrm{~m}^{-1}\left(k_{x}=1.0 \mathrm{~m}^{-1}, k_{y}=1.0 \mathrm{~m}^{-1}\right)$.

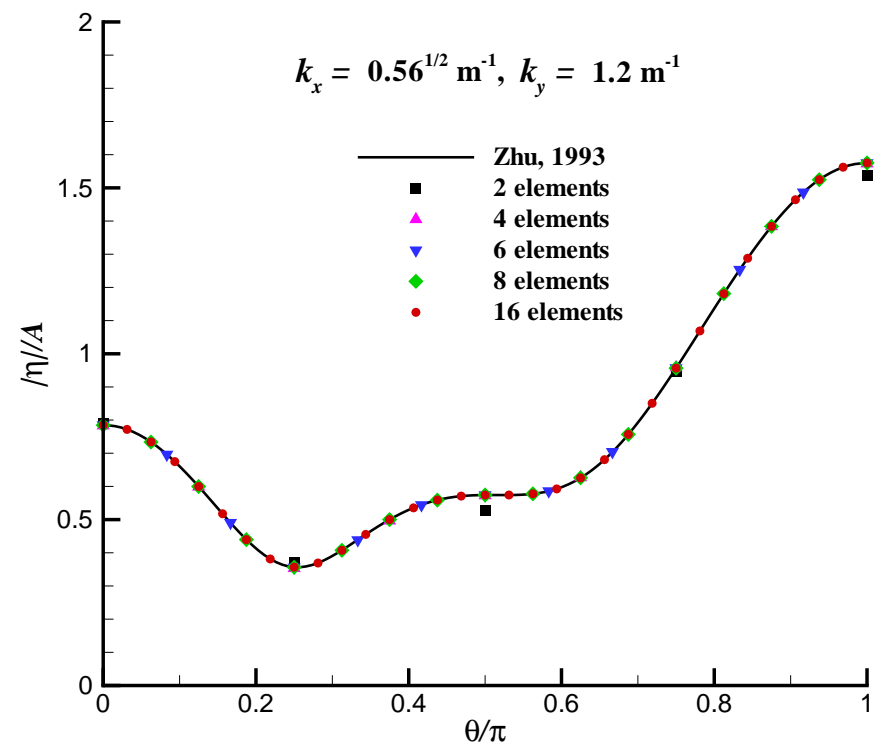

Fig. 10. Comparison of short-crested wave run-up on a cylinder with radius $a=1.0 \mathrm{~m}$ and total incident wave number $k=\sqrt{2} \mathrm{~m}^{-1}\left(k_{x}=\sqrt{0.56} \mathrm{~m}^{-1}, k_{y}=1.2 \mathrm{~m}^{-1}\right)$. 


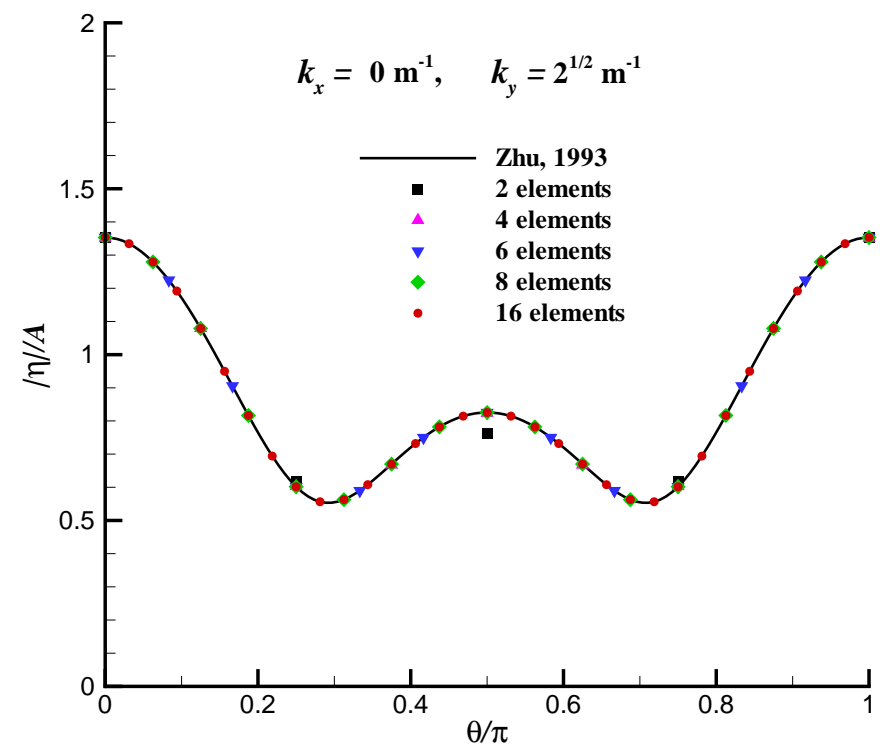

Fig. 11. Comparison of short-crested wave run-up on a cylinder with radius $a=1.0 \mathrm{~m}$ and total incident wave number $k=\sqrt{2} \mathrm{~m}^{-1} .\left(k_{x}=0 \mathrm{~m}^{-1}, k_{y}=\sqrt{2} \mathrm{~m}^{-1}\right)$

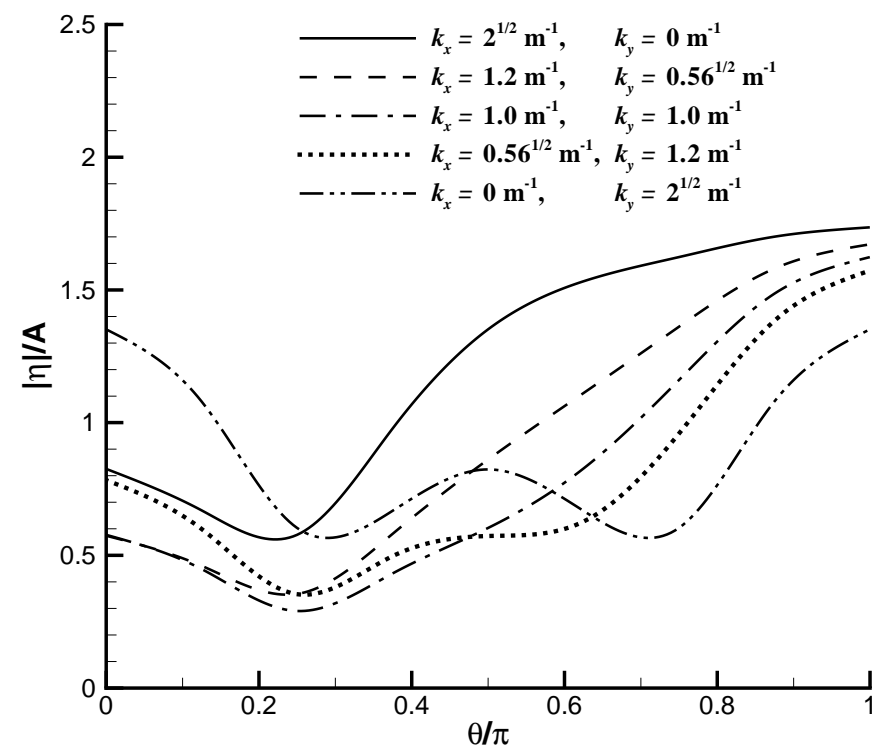

Fig. 12. Wave run-up on a cylinder with radius $a=1.0 \mathrm{~m}$ and total incident wave number $k=\sqrt{2} \mathrm{~m}^{-1}$ : Plane waves vs. Short-crested waves. 
264 as can be seen in Table 1 , for a fixed $k a, C_{M}$ and $C_{D}$ are clearly unchanged for all 265 the cases with the variation of $k_{x}$ and $k_{y}$. It should be noted that the digits with the 266 superscript * are reversed in [17] and the digits with the superscript ${ }^{\#}$ are erroneous 267 in [17]. 


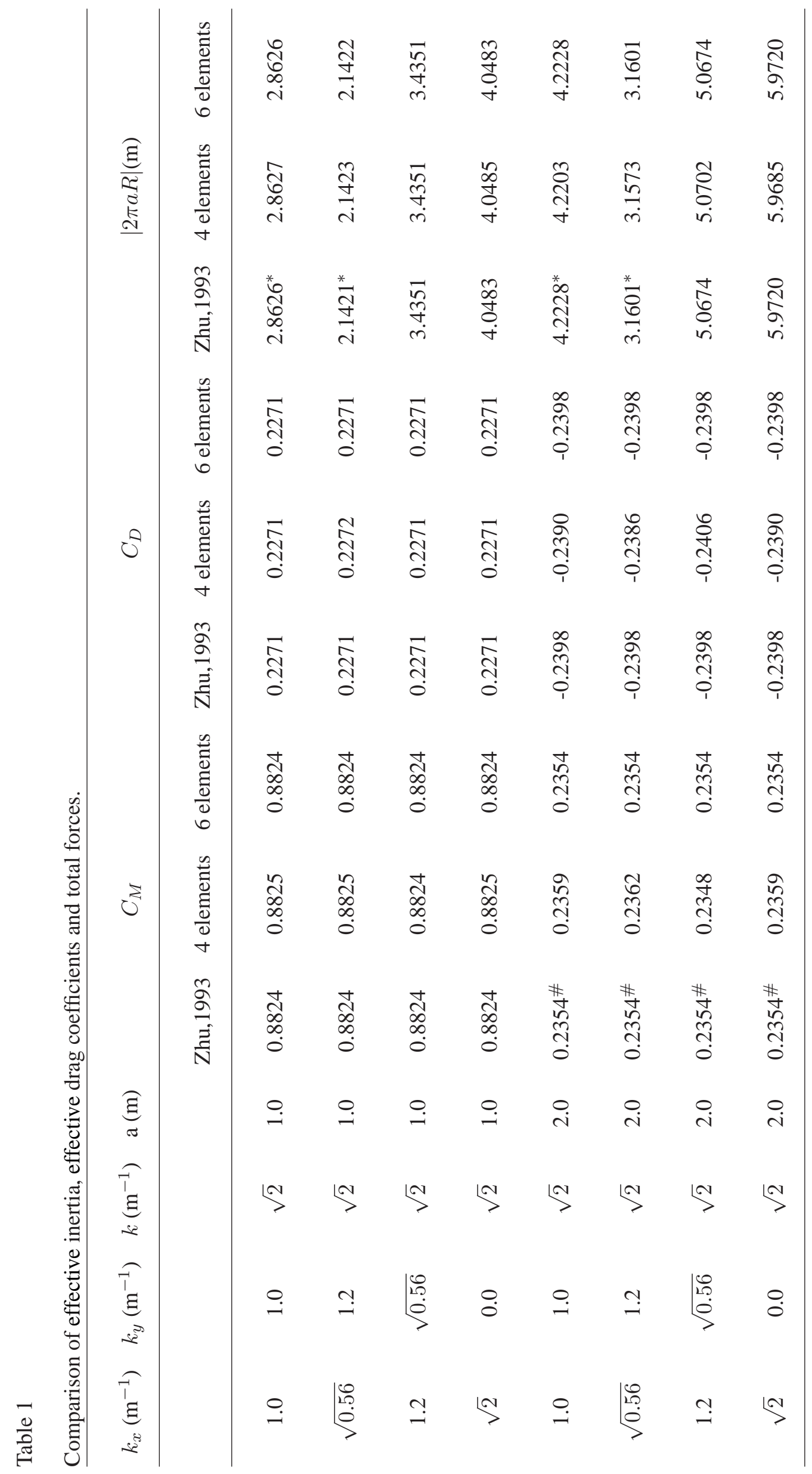


Further calculations revealed that $C_{M}$ and $C_{D}$ per unit length are invariants for a fixed $k a$. To demonstrate this, the variation of $C_{M}$ and $C_{D}$ are plotted against the variation of the ratio $k_{y} / k_{x}$ in Figs. 13 and 14, for four different values of $k_{x}$, while $k_{x} a$ held as a constant, corresponding to the following cases: 1) $k_{x}=0.8 \mathrm{~m}^{-1}$, $a=2.5 \mathrm{~m}$; 2) $k_{x}=1.0 \mathrm{~m}^{-1}, a=2.0 \mathrm{~m}$; 3) $k_{x}=1.6 \mathrm{~m}^{-1}, a=1.25 \mathrm{~m}$; 4) $k_{x}=2.0 \mathrm{~m}^{-1}, a=1.0 \mathrm{~m}$. As can be clearly seen in the figures, four curves coincide with each other. This indicates that coefficients $C_{M}$ and $C_{D}$ are invariants of $k a$. This attribute was also shown in [17], but with the misprint of $k_{x}$ shown as $k$ in the text as well as in the legend. This is not a surprising result as a short-crested wave can occur from the oblique interaction of two travelling plane-waves, thus linear forces from short-crested wave interaction with a cylinder can be effectively obtained by the superposition of two plane-waves. Therefore coefficients $C_{M}$ and $C_{D}$ are physically invariants of $k a$. However, the total force $|2 \pi a R|$ for the above four cases are different, as shown in Fig. 15, the total force decreases as the incident wave becomes more short-crested. It is noted that all the SBFEM results presented in Figs. 13-15 are calculated with 6 discretized elements on the cylinder boundary.

Figs. 16-21 show the co-amplitude and co-phase lines for long- and short-crested incident waves diffracted by a circular cylinder with a radius of $1.0 \mathrm{~m}$. The amplitude is nondimensionalized as $|\eta| / A$ and the phases are between $[-\pi \sim+\pi]$. $k_{x}$ is fixed as $k_{x}=1.0 \mathrm{~m}^{-1}$, and $k_{y}$ has different values: $0,0.5$, to $1.0 \mathrm{~m}^{-1}$ respectively. It is apparent that the wave patterns of diffracted short-crested waves are more complicated. The thick lines in all the co-phase plots show the sudden change of the phase values from $-\pi$ to $\pi$. From these results, one can easily draw the conclusion that as the incident waves become more short-crested, the amplitude of the diffracted waves in the rear region become smaller and the region of the co-amplitude line upstream reduces. The amphidromic points come forth for short- 


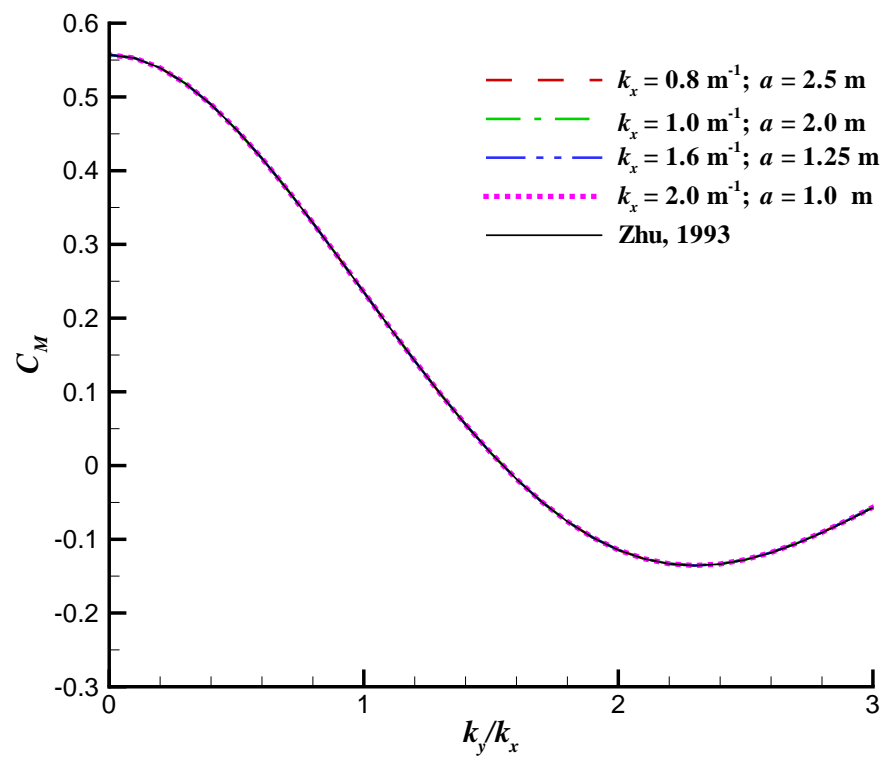

Fig. 13. Variation of the effective inertial coefficient $C_{M}$ vs the ratio $k_{y} / k_{x}$ at $k_{x} a=2$.

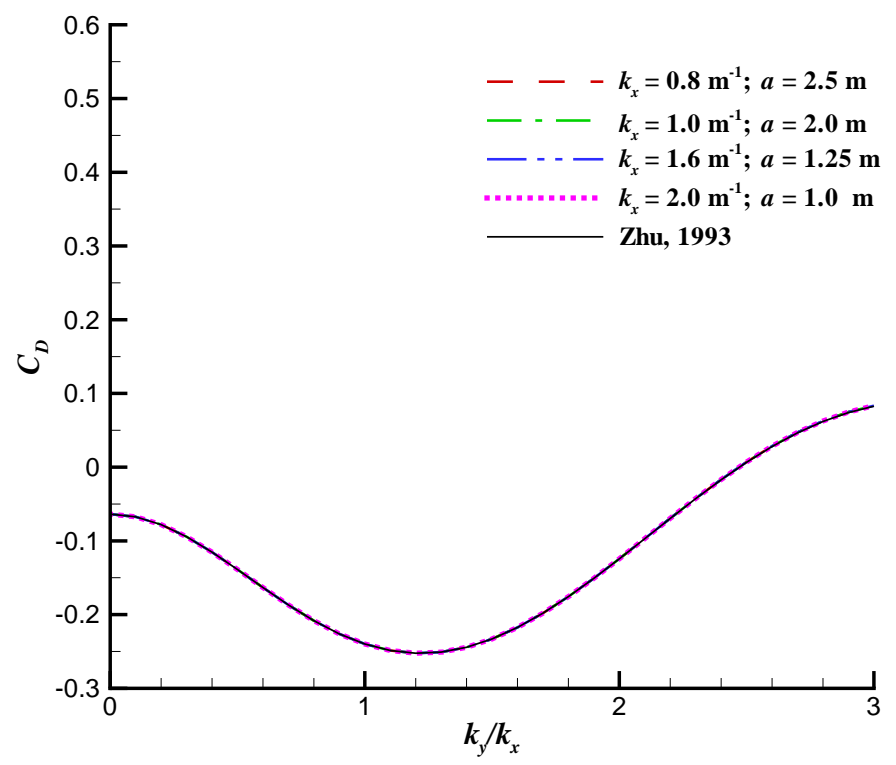

Fig. 14. Variation of the effective drag coefficient $C_{D}$ vs the ratio $k_{y} / k_{x}$ at $k_{x} a=2$. waves become more short-crested. 


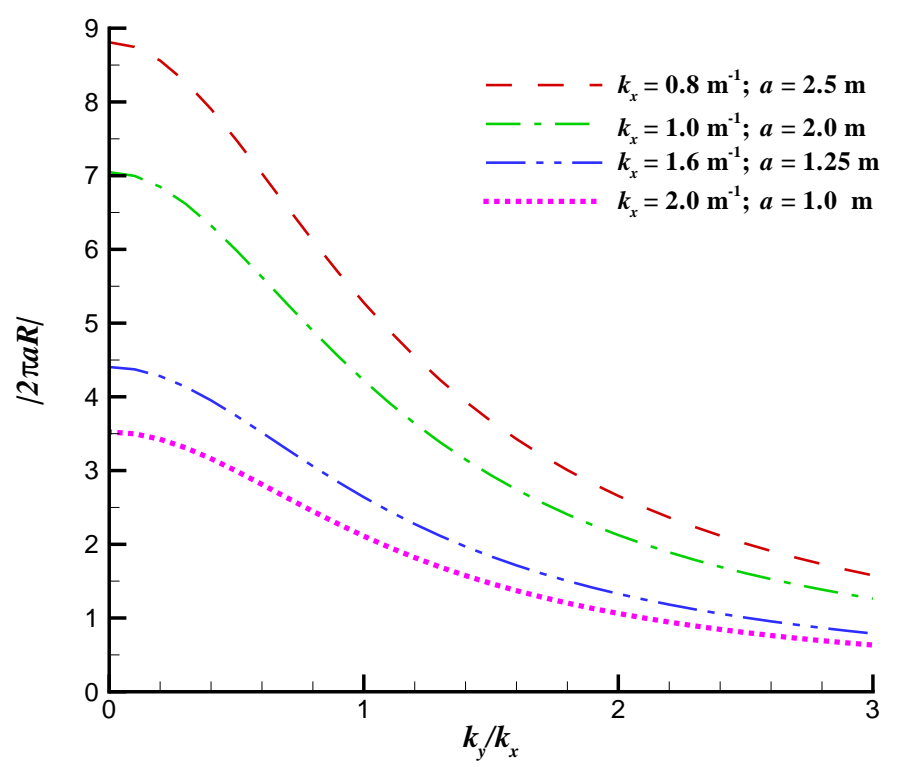

Fig. 15. Variation of the total force vs the ratio $k_{y} / k_{x}$ at $k_{x} a=2$.

It is worth noting that, the computation times (recorded on a $2 \mathrm{GHz}$ Pentium IV PC and MATLAB 7.1) of the scaled boundary finite-element method solutions are very small. For all the cases presented in this paper, accurate results are obtained in less than $3 \mathrm{~s}$, a clear demonstration that it significantly outperforms any current finiteelement or boundary-element method for similar problems. Such computational efficiency and accuracy ensure great potential of direct application of the present method to many engineering problems especially in ocean engineering.

\section{Conclusions}

The semi-analytical scaled boundary finite-element method has been successfully applied to solve the diffraction of short-crested waves incident on a circular cylinder. In contrast to the conventional boundary-element method, which has been widely applied to wave-structure interaction problem in unbounded domain, the 


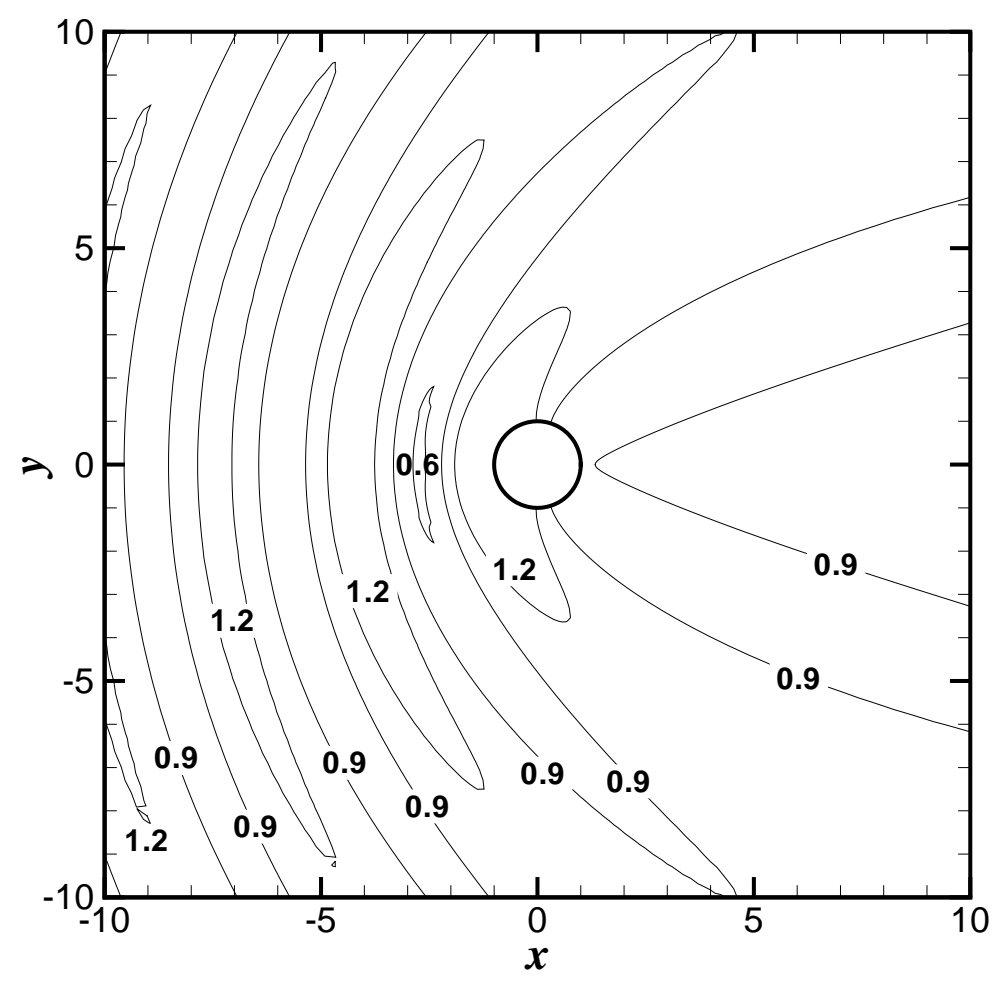

Fig. 16. The curve of equal amplitude (co-amplitude) for the incident waves with longitudinal and lateral wave numbers $k_{x}=1.0 \mathrm{~m}^{-1}$ and $k_{y}=0.0 \mathrm{~m}^{-1}$, respectively.

new technique requires no help from any fundamental solutions. A reduction of one in the spatial dimension is achieved with this procedure, since only the body boundary is discretized with surface finite-elements. Excellent computational efficiency and accuracy of the present scaled boundary finite-element method has been demonstrated, as the governing equations are solved analytically in the radial direction. In solving the short-crested wave problem, the present numerical method is shown to reproduce the analytical solution for all the physical properties including wave run-up, effective inertia and drag force coefficients, and total force very accurately and at very low computational cost. The method holds promise in solving more practical ocean engineering problems. 


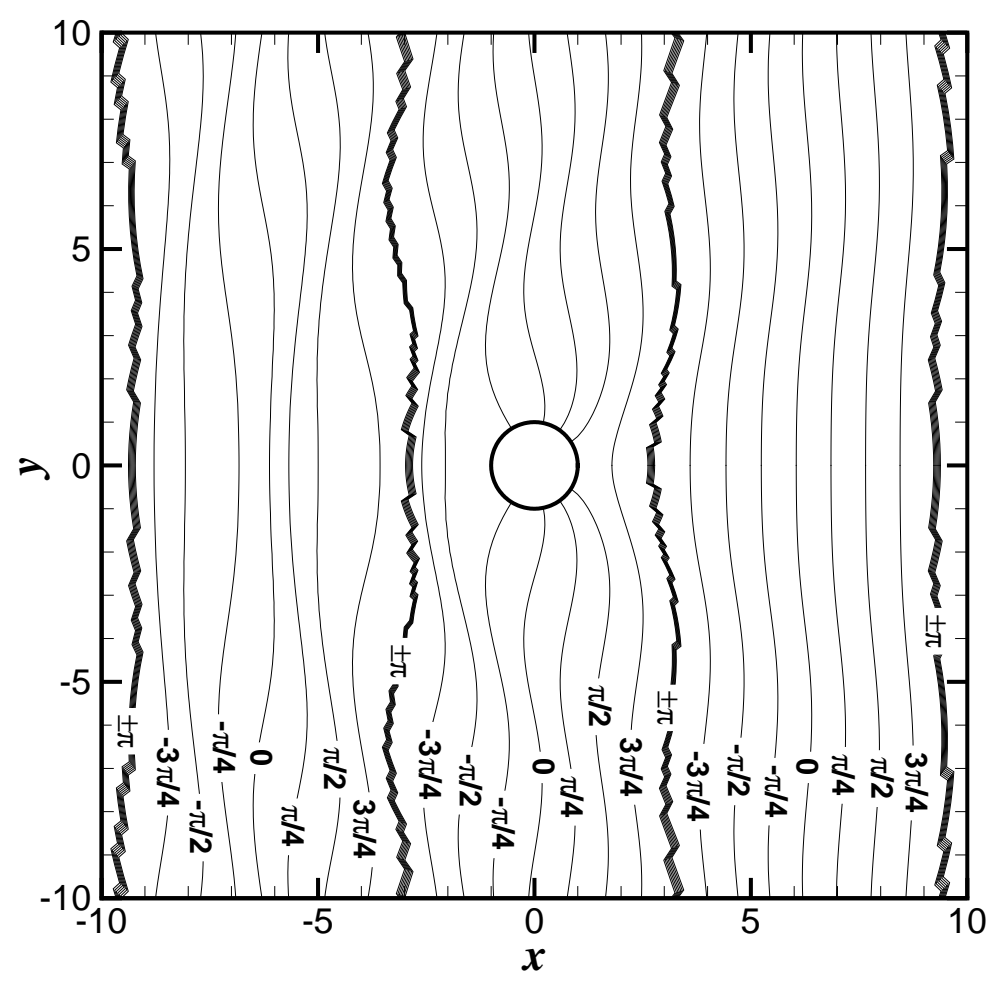

Fig. 17. The curve of equal phase (co-phase) for the incident waves with longitudinal and lateral wave numbers $k_{x}=1.0 \mathrm{~m}^{-1}$ and $k_{y}=0.0 \mathrm{~m}^{-1}$, respectively.

\section{Acknowledgment}

\section{References}

[1] M.C. Au, C.A. Brebbia, Diffraction of water waves for vertical cylinders using boundary elements, Appl. Math. Model. 7 (2) (1983) 106-114.

[2] S.K. Chakrabarti, W.A. Tam, Interaction of waves with large vertical cylinder, J. Ship 


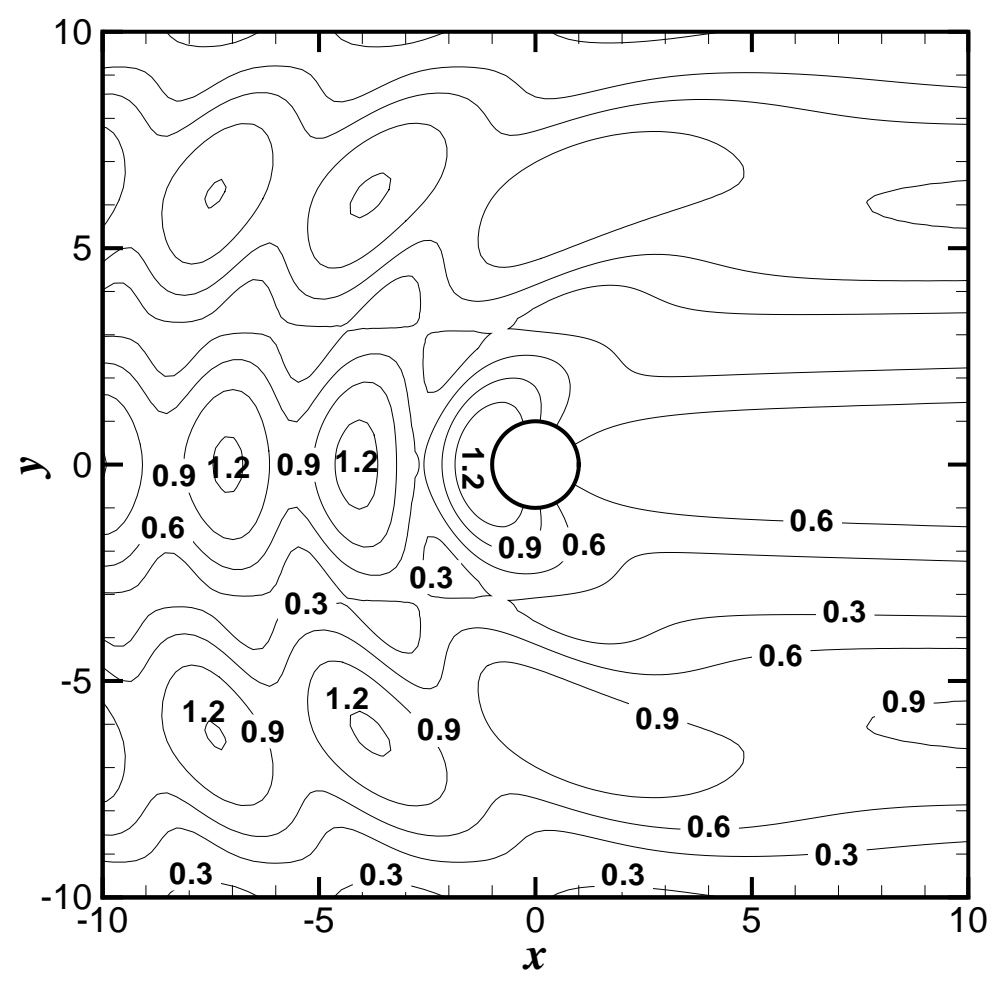

Fig. 18. The curve of equal amplitude (co-amplitude) for the incident waves with longitudinal and lateral wave numbers $k_{x}=1.0 \mathrm{~m}^{-1}$ and $k_{y}=0.5 \mathrm{~m}^{-1}$, respectively. Res. 19 (1) (1975) 23-33.

[3] S.K. Chakrabarti, Steady drift force on vertical cylinder - viscous vs. potential, Appl. Ocean Res. 6 (2) (1984) 73-82.

[4] A.J. Deeks, L. Cheng, Potential flow around obstacles using the scaled boudary finiteelement method, Int. J. Numer. Meth. Fluids 41 (2003) 721-741.

[5] R.A. Fuchs, On the theory of short-crested oscillatory waves, Gravity Waves, National Bureau of Standards Circular No. 521, Department of Commerce, USA, (1952) 187200.

[6] D.R. Fuhrman, P.A. Madsen, Short-crested waves in deep water: a numerical investigation of recent laboratory experiments, J. Fluid Mech. 559 (2006) 391-411.

[7] D. Lesnic, L. Elliott, D.B. Ingham, Boundary element methods for determining the 


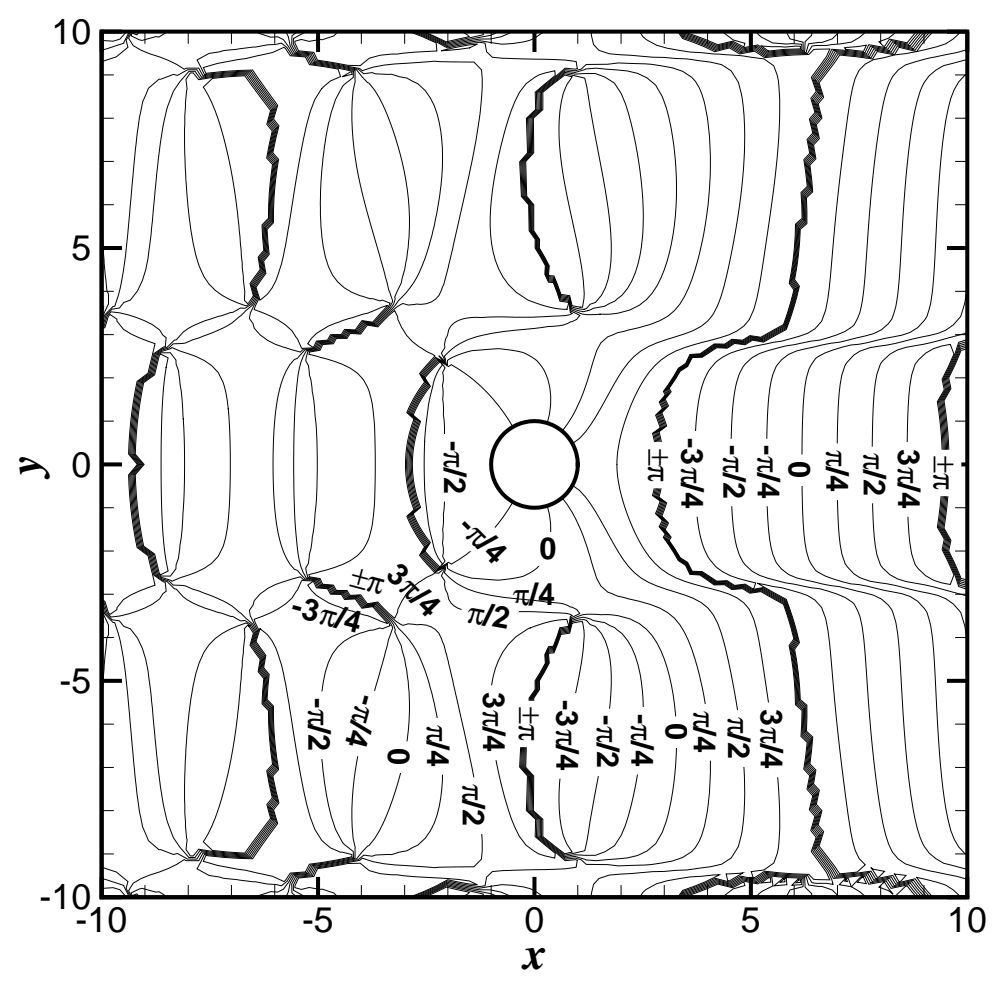

Fig. 19. The curve of equal phase (co-phase) for the incident waves with longitudinal and lateral wave numbers $k_{x}=1.0 \mathrm{~m}^{-1}$ and $k_{y}=0.5 \mathrm{~m}^{-1}$, respectively. fluid velocity in potential flow, Eng. anal. bound. elem. 11 (3) (1993) 203-213.

[8] B. Li, L. Cheng, A.J. Deeks, Wave diffraction by vertical cylinder using the scaled boudary finite element method, WCCM VI \& APCOM'04, Beijing, China, 2004.

[9] P.A. Martin, A.G. Dixon, The scattering of regular surface waves by a fixed, halfimmersed, circular cylinder, Appl. Ocean Res. 5 (1) (1983) 13-23.

[10] R.C. MacCamy, R.A. Fuchs, Wave forces on piles: a diffraction theory, Tech. Memo. No. 69, U.S. Army Board, U.S. Army Corp. of Eng. 1954.

[11] C.C. Mei, The applied dynamics of ocean surface waves, World Scientific, Singapore, 1989.

[12] S. Neelamani, V. Sundar, C.P. Vendhan, Dynamic pressure distribution on a cylinder due to wave diffraction, Ocean Eng. 16 (4) (1989) 343-353. 


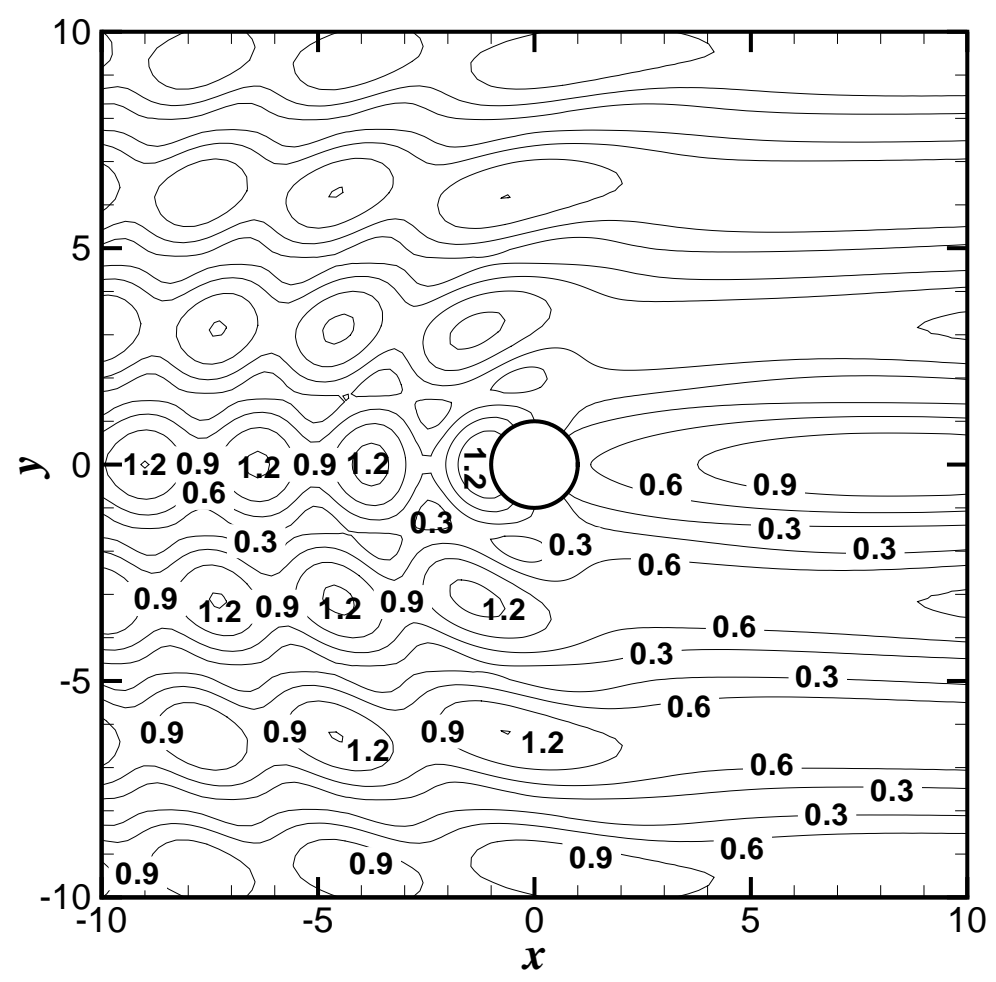

Fig. 20. The curve of equal amplitude (co-amplitude) for the incident waves with longitudinal and lateral wave numbers $k_{x}=1.0 \mathrm{~m}^{-1}$ and $k_{y}=1.0 \mathrm{~m}^{-1}$, respectively.

[13] V. Sundar, S. Neelamani, C.P. Vendhan, Diffracted wave field and dynamic pressures

[18] S. Zhu, G. Moule, Numerical calculation of forces induced by short-crested waves on 


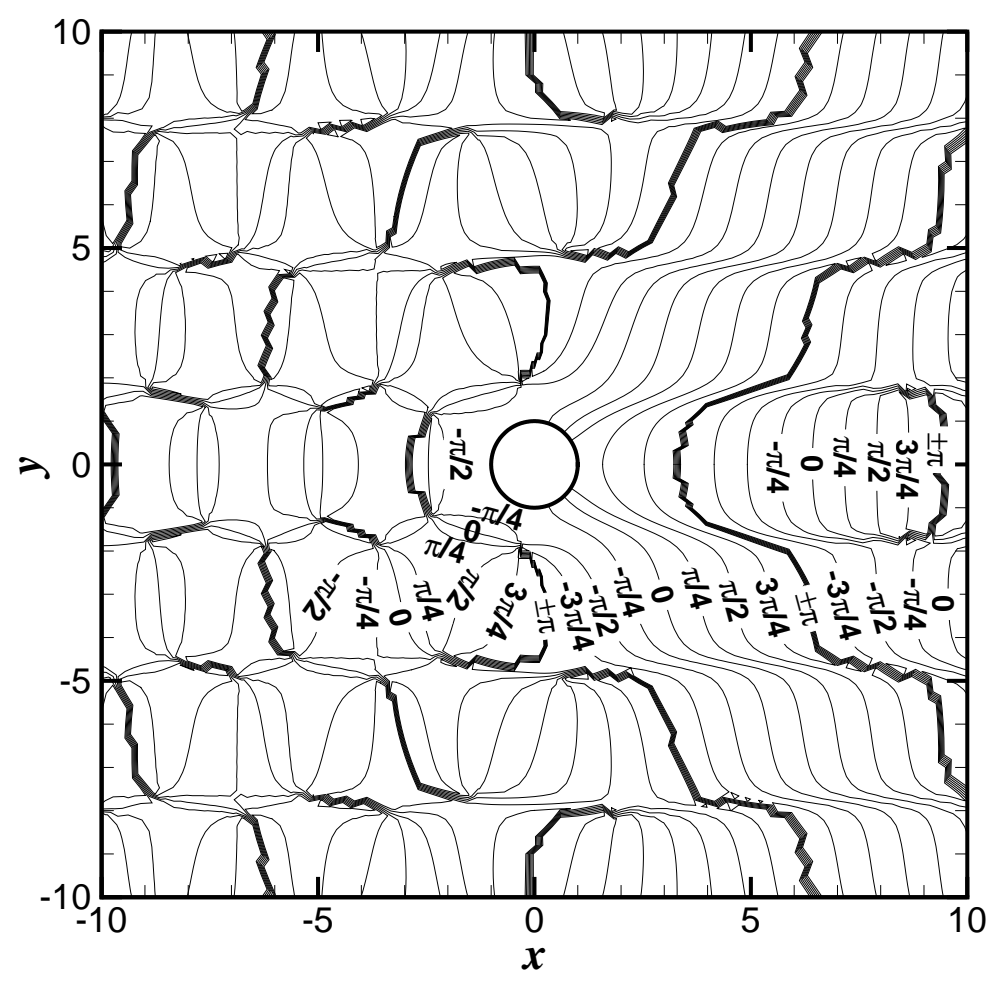

Fig. 21. The curve of equal phase (co-phase) for the incident waves with longitudinal and lateral wave numbers $k_{x}=1.0 \mathrm{~m}^{-1}$ and $k_{y}=1.0 \mathrm{~m}^{-1}$, respectively. a vertical cylinder of arbitrary cross-section, Ocean Eng. 21 (7) (1994) 645-662.

[19] S. Zhu, P. Satravaha, Second-order wave diffraction forces on a vertical circular cylinder due to short-crested waves, Ocean Eng. 22 (2) (1995) 135-189. 